\section{Casein Kinase 1 Modulator PF-05251749}

Circadian rhythm is regulated by the body's inner central clock. This clock controls key body functions, including wake/ sleep cycles, body temperature, digestion, hormonal cycles, and behavioural patterns. Patients afflicted with Parkinson disease often show symptoms of a condition collectively called irregular sleep wake rhythm disorder. Irregular sleep wake rhythm disorder is a circadian rhythm disorder where patients experience fragmented night-time sleep leading to daytime sleepiness, severe fatigue and difficulty with activities of daily living. Another symptom associated with sleep-wake cycle is sundowning. It affects $20 \%$ or more patients diagnosed with Alzheimer's disease, who become confused, anxious, aggressive, agitated or restless later in the day. It usually increases in frequency over the course of the disease and disability progression [1].

Many drugs, primarily psychopharmaceuticals, target one or more aspects of the sleepwake cycle in order to normalise circadian rhythm, but still do so with quite a number of side effects or adverse reactions. Therefore, it comes as no surprise that fine-tuning of the circadian rhythm emerged as an interesting research target. In light of that fact, casein kinase 1 modulators seem to be at the forefront of that research, especially a compound, just known as PF-05251749. It is a regulator of the body's inner clock, which is a part of a group of potential disease-modifying therapies for Parkinson's and Alzheimer's diseases. Namely, a small region within the brain's hypothalamus - the suprachiasmatic nucleus is responsible for controlling circadian rhythms, and an enzyme called casein kinase 1 (CK1), which exists in two forms, is a key regulator of this clock. CK1 epsilon and CK1 delta are closely related Ser-Thr protein kinases and the fact that they serve as key clock regulators is demonstrated by dramatic alterations of the circadian period in mammals with mutations of those proteins. Specifically, genetic ablation of CK1 delta alters the length of a single cycle of sleep-wake, and loss of CK1 epsilon results in arrhythmicity.

Biogen Inc. recently acquired PF05251749 and will test it further in clinical trials as a treatment to correct irregular sleep wake rhythm disorder in Parkinson's disease and sundowning in Alzheimer's disease [2]. Pfizer Corporation primarily set to devel- 
op this compound for the treatment of $\mathrm{Al}$ zheimer's disease, but it was discontinued due to Pfizer's ending of its neurology research and development [3].

Investigated compound PF-05251749 is an oral, brain-penetrable, highly selective, inhibitor of both forms of CK1. Inhibiting CK1 disrupts the function of two genes, Circadian locomotor output cycles kaput (CLOCK) and Brain and Muscle ARNT-Like 1 (BMAL1), which are thought to influence the regulation of circadian rhythms [2]. The chemical structure of the compound is yet not disclosed. PF-05251749 has previously demonstrated an acceptable safety profile in Phase Ia clinical studies. Those consisted of two Phase 1 clinical trials in healthy volunteers, aged 18 to 55 and 18 to 85 . They were conducted to determine not only the safety of PF-05251749 but also to assess pharmacokinetics and pharmacodynamics, as well as to find out how much of the compound reaches the cerebrospinal fluid. Biogen Inc. aims to initiate a Phase Ib study in fourthquarter of 2020 [4,5].

Alzheimer's disease and Parkinson's disease are characterized with debilitating sleep disorders and agitation. Therefore, regulation of the circadian rhythm might hold promise to addressing these behavioural and neurological symptoms. One of major targets are CK1 modulators, as CK1 belongs to a large family of protein kinases that function as regulators of signal transduction pathways in most eukaryotic cell types. Different CK1 inhibitors are currently in various stages of research, such as D4476, PF-670462, PF4800567 hydrochloride and others [6]. Since this is a drug class with a new mechanism of action, along with a significant clinical potential, we look forward to their further development and research updates.

\section{Assistant Professor Vjekoslav Peitl, MD, PhD Darko Vlahović, $M D$}

\section{References}

1. Biogen news. Biogen to acquire novel clinical stage asset with application in Alzheimer's disease and Parkinson's disease from Pfizer Inc. [Internet]. 2020 [cited March 20th] Available from: https:// investors.biogen.com/news-releases/news-release-details/biogen-acquire-novel-clinical-stageasset-application-alzheimers.

2. Inacio P. Biogen Acquires Pfizer's PF-05251749, a Potential Disease-modifying Therapy for Parkinson's, Alzheimer's [Internet]. 2020 [cited March 20th] Available from: https://parkinsonsnewstoday.com/2020/01/15/biogen-pfizerpf-05251749-potential-disease-modifying-therapy-parkinsons-alzheimers/.
3. Alzforum database of therapeutics. Overview of PF-05251749 [Internet]. 2020 [cited March 20th] Available from: https://www.alzforum.org/therapeutics/pf-05251749.

4. Thepharmaletter. Biogen reinforces bet on Alzheimer's, buying in Pfizer CNS candidate [Internet]. 2020 [cited March 20th] Available from: https://www.thepharmaletter.com/article/biogen-reinforces-bet-on-alzheimer-s-buying-in-pfizer-cns-candidate.

5. Parkinson's news today. PF-05251749 [Internet]. 2020 [cited March 20th] Available from: https:// parkinsonsnewstoday.com/pf-05251749/

6. R\&D Systems. Casein Kinase 1 Inhibitors: Products [Internet]. 2020 [cited March 20th] Available from: https://www.rndsystems.com/products/ pf-670462_3316 